\title{
Optical and Structural Properties of Bilayer Circular Filter Prepared by Glancing Angle Deposition
}

\author{
Yong Jun Park, KM Abdus Sobahan, Jin Joo Kim, and Chang Kwon Hwangbo* \\ Department of Physics, Inha University, Incheon 402-751, Korea
}

(Received December 8, 2008 : accepted February 3, 2009)

\begin{abstract}
In this paper, we report the optical and structural properties of a bilayer circular filter fabricated by a glancing angle deposition technique. The bilayer circular filter is realized by a two-layer $\mathrm{TiO}_{2}$ helical film with layers of opposite structural handedness. It is found that the bilayer circular filter reflects both right and left circularly polarized light with wavelength lying in the Bragg regime. The microstructure of the bilayer circular filter is also investigated using a scanning electron microscope.
\end{abstract}

Keywords: Helical film, Glancing angle deposition, Bragg reflector

OCIS codes : (310.0310) Thin films; (310.1860) Deposition and fabrication; (310.6860) Thin films, optical properties; (230.1480) Bragg reflectors

\section{INTRODUCTION}

Sculptured thin films (STFs) are nanoengineered materials with unidirectionally varying properties that can be designed and realized in a controllable manner using physical vapor deposition. In the recent years, STFs become more valuable as a platform of optical devices to the optics community [1-4]. Glancing angle deposition (GLAD), an advanced technique for fabricating designed microstructures at nanometer scale, is used to fabricate the STFs. In this technique, oblique angle deposition and substrate rotation are employed to control the microstructures of the films. By exploiting various rotation schemes for the substrate, a wide variety of thin-film morphologies including slanted columns, helices, S-shapes, C-shapes and zigzag/chevron structures can be fabricated [5-8]. Because of controlled porosity and microstructure, GLAD films have a wide range of suggested applications, such as three-dimensional photonic crystals [9-11], birefrigent omnidirectional reflectors [12], graded index optical filters [13, 14], broadband antireflection coatings [15, 16], linear polarizers [17], and fluid concentration sensors $[18,19]$.

Any chiral STFs, simply referred to as helical films, exhibit the so-called circular Bragg phenomenon upon axial excitation by a plane wave due to the periodic and rotational variation of its constitutive properties along a fixed axis, i.e., it will preferentially reflect circularly polarized light of the same handedness, while transmitting circularly polarized light of the opposite handedness $[1,20]$. This statement is supported by observations on both chiral liquid crystals [21] and chiral STFs [22]. Due to this ability to discriminate between left and right circularly polarized light, any helical film can be used for circular polarization elements including sources, reflectors, filters, and detectors [23-26]. The peak wavelength, $\left(\lambda_{0}{ }^{B r}\right)$ of the Bragg regime is proportional to both the average refractive index, $n_{a \nu}$ as well as film half-pitch thickness, $\Omega$ and is calculated by using the equation, $\lambda_{0}{ }^{B r}=2 \Omega n_{a} \nu$ $[27,28]$.

The aim of the present work is to study the optical and structural properties of a bilayer circular filter realized by a two-layer $\mathrm{TiO}_{2}$ helical film with layers of opposite structural handednesses. The Bragg effect of a $\mathrm{TiO}_{2}$ helical film with different structural handednesses is also studied. These devices are fabricated by electron-beam evaporation using the GLAD technique. The optical properties and microstructures of the devices are characterized by using a spectrophotometer and a scanning electron microscope.

*Corresponding author: hwangbo@inha.ac.kr 


\section{EXPERIMENTAL}

The electron-beam evaporation with the GLAD technique was used to fabricate the helical films and the bilayer circular filter. The simplified schematic diagram of the GLAD technique is shown in Fig. 1. The deposition was performed in a vacuum chamber with a base pressure of $\sim 5 \times 10^{-6}$ Torr. The electron-beam evaporator with a $3-\mathrm{cm}$ crucible pocket was located at $45 \mathrm{~cm}$, directly beneath the substrate. The deposition rate and thickness of the growing films were measured by a quartz-crystal sensor, which was placed near the substrate. Glass (B270, $70 \mathrm{~mm} \times 50 \mathrm{~mm} \times 1 \mathrm{~mm}$ ) and polished $\mathrm{Si}(100)$ wafers were used as substrates. Vapor flux incident angle, $60^{\circ}$ and substrate rotation speed, $0.09 \mathrm{rpm}$ were employed to control the columnar microstructures of the films. The deposition rate of the films was kept at $0.5 \mathrm{~nm} / \mathrm{s}$ by using a quartz-crystal sensor. The devices deposited on the glass substrates were used for optical analysisand those of deposited on silicon wafers were used for structural analysis. The transmittance spectra of the devices were measured by a spectrophotometer (Cary 500, Varian) in the wavelength range of 400 to $1100 \mathrm{~nm}$.

A linear polarizer, achromatic quarter-wave plate, sample, and another achromatic quarter-wave plate as well as another linear polarizer were placed in the beam path of a spectrophotometer to measure the transmittance spectra of the devices. Left circular polarized (LCP) and right circular polarized (RCP) light was generated by passing unpolarized light through a linear polarizer followed by an achromatic quarter-wave plate with its fast axis oriented $\pm 45^{\circ}$ relative to the transmission axis of the linear polarizer. The cross-sectional image of the devices was investigated using a scanning electron microscope (SEM).

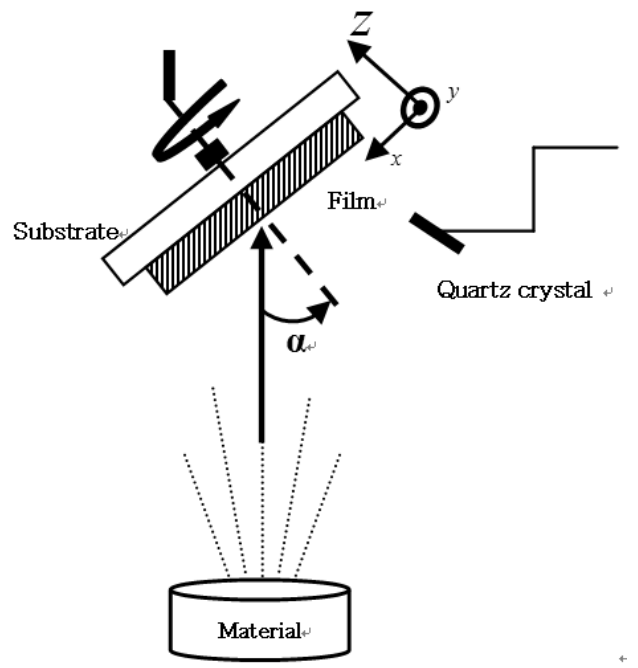

FIG. 1. Schematic diagram of glancing angle deposition.

\section{RESULTS AND DISCUSSION}

\section{OPTICAL PROPERTIES}

Figure 2 shows the measured reflectance spectra of 5-turns $\mathrm{TiO}_{2}$ helical films with different structural handednesses. The thickness of one turn, called pitch thickness, is equal to $380 \mathrm{~nm}$. In Fig. 2 (a), the $\mathrm{TiO}_{2}$ helical film with left-handed structure reflects the left circular polarized light within the Bragg regime. A similar nature is found in the case of right-handed structural film in which the right circular polarized light within the Bragg regime is reflected, as illustrated in Fig. 2 (b). The result assures that the $\mathrm{TiO}_{2}$ helical films preferentially reflect circularly polarized light of the same handedness, while transmitting circularly polarized light of the opposite handedness. Theses spectra carry the signature of the circular Bragg phenomenon with peak wavelength, 650 $\mathrm{nm}$ in the Bragg regime, which occurs due to unidirectionally periodic nonhomogeneity and helical morphology along the thickness direction. This phenomenon can also be explained by using grating theory [29]: a circular

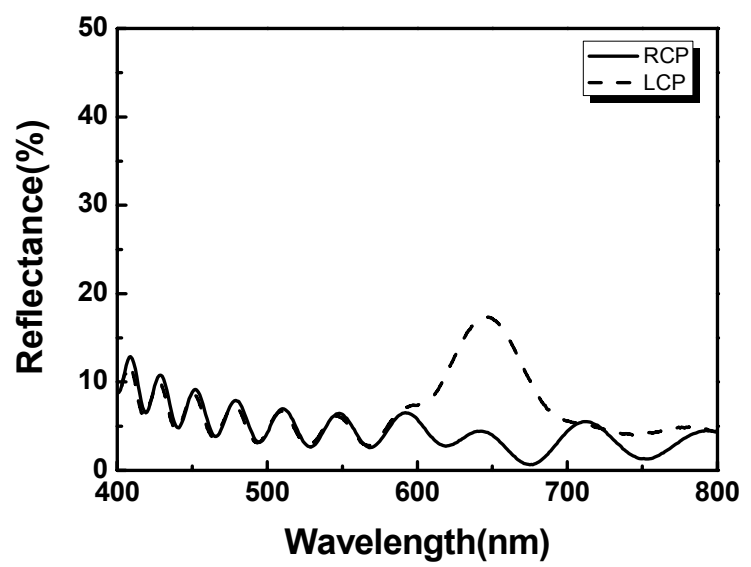

(a)

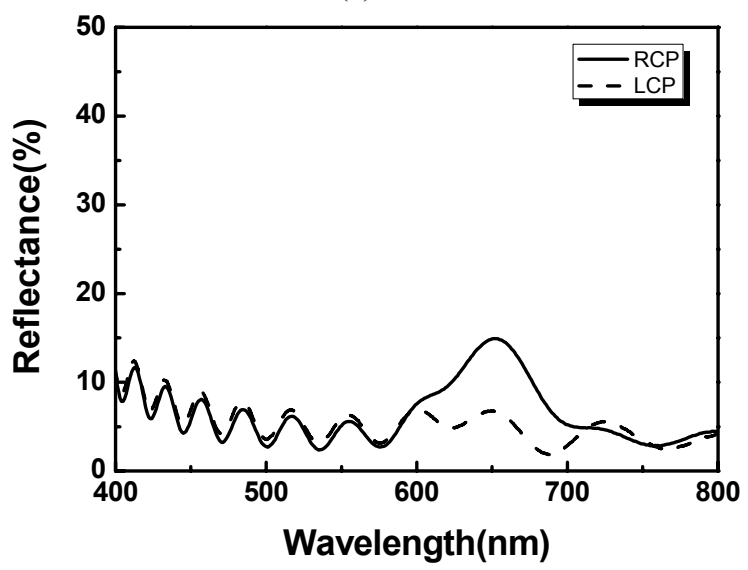

(b)

FIG. 2. Reflectance spectra of 5-turns $\mathrm{TiO}_{2}$ helical films deposited at $60^{\circ}$ with different structural handednesses: (a) left $(\mathrm{H}=-1)$ and (b) right $(\mathrm{H}=+1)$. 
plane wave of the same handedness effectively encounters the Bragg grating, while that of the other handedness does not. The peak wavelengths in the Bragg regime of these films are almost the same due to fixed pitch thickness and deposition angle [30].

The circular Bragg phenomenon is exploited to fabricate and test the bilayer circular filter realized by the two-layer $\mathrm{TiO}_{2}$ helical film of opposite structural handednesses. Figure 3 presents the reflectance and the selective transmittance $(\mathrm{LCP}-\mathrm{RCP})$ of the bilayer circular filter. It is clear that the bilayer circular filter reflects both circular polarizations of light (LCP \& RCP) with wavelength lying in the Bragg regime. This happens due to the opposite structural handedness of the bilayer filter. In Fig. 3 (a), the peak reflectance for both LCP and RCP light is found to be approximately $27 \%$ at a peak wavelength of $825 \mathrm{~nm}$ in the Bragg regime. The selective transmittance ( $\mathrm{LCP}-\mathrm{RCP}$ ) of this filter is also close to zero, as shown in Fig. 3 (b). These results indicate that the bilayer circular filter is clearly a polarization -insensitive bandstop filter.

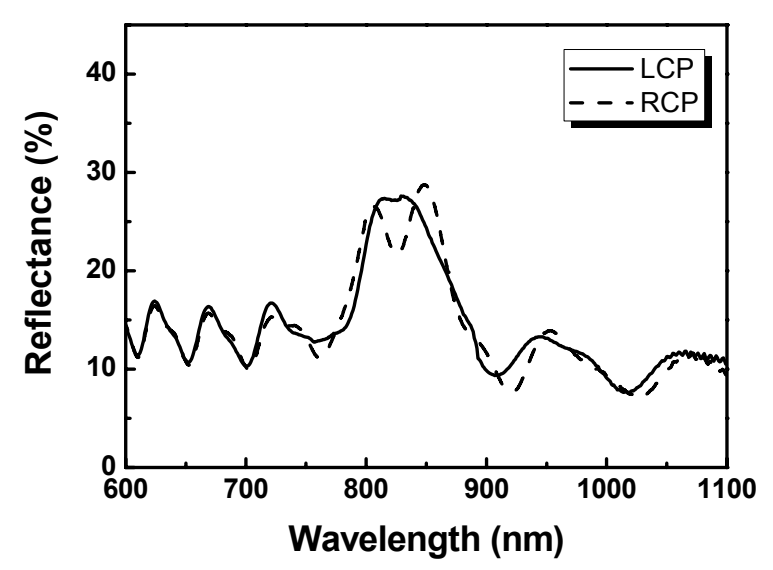

(a)

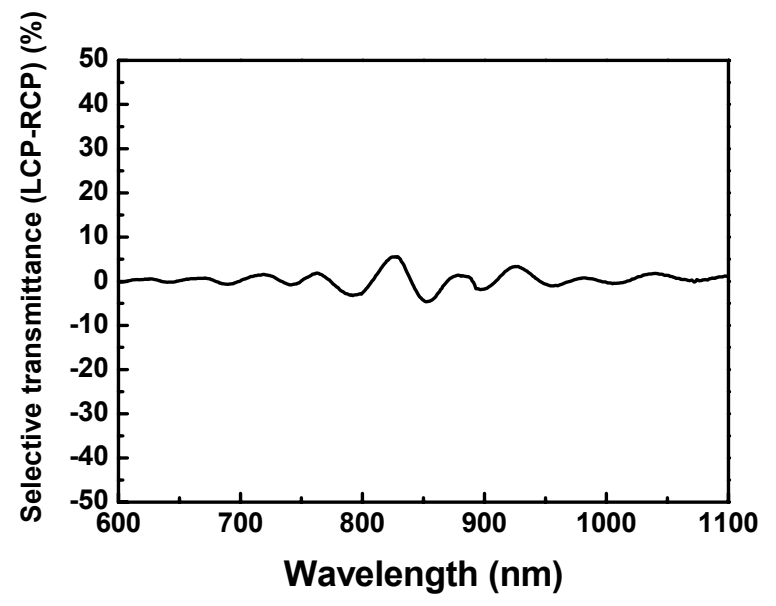

(b)

FIG. 3. Remittance of bilayer circular filter: (a) reflectance and (b) selective transmittance ( $\mathrm{LCP}-\mathrm{RCP}$ ).

\section{STRUCTURAL PROPERTIES}

The cross-sectional SEM image of the $\mathrm{TiO}_{2}$ helical films with different structural handednesses and the bilayer circular filter of opposite structural handednesses are illustrated in Fig. 4. Figures 4 (a) and (b) depict the 5-turns left-handed and right-handed structures of the $\mathrm{TiO}_{2}$ helical films, respectively. The thickness of each $\mathrm{TiO}_{2}$ helical film is $1.9 \mu \mathrm{m}$ and the center axis of the

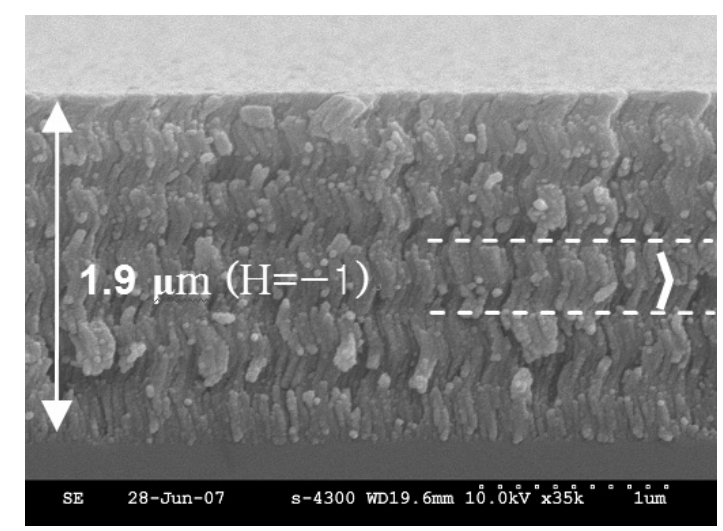

(a)

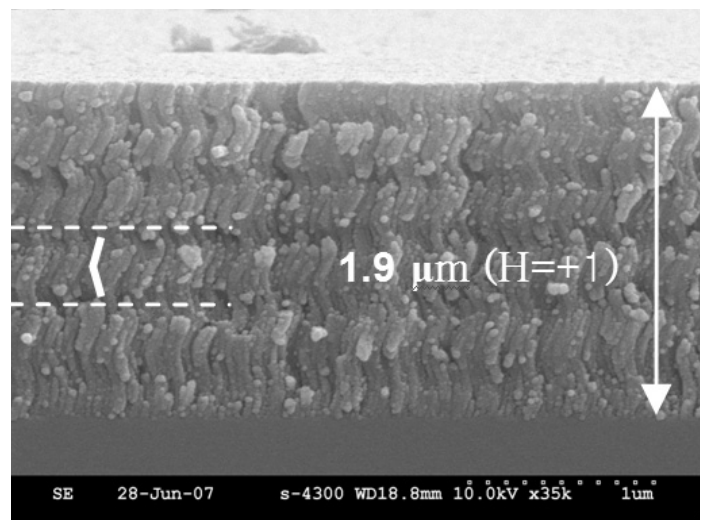

(b)

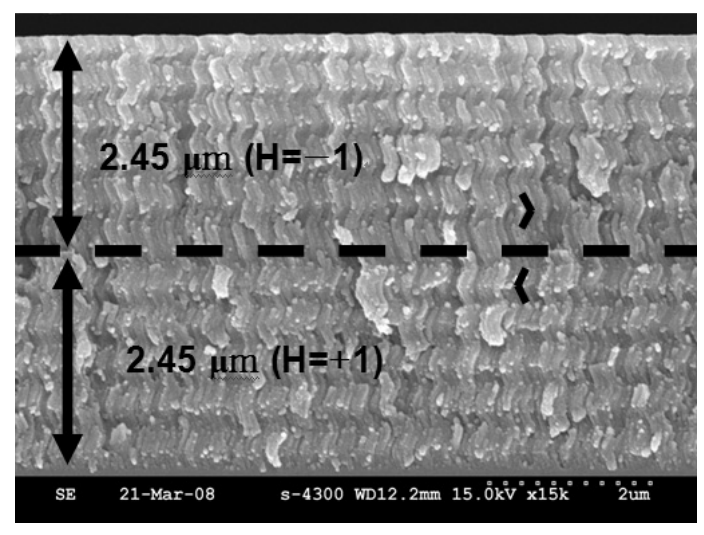

(c)

FIG. 4. Cross-sectional SEM images: (a) 5-turns lefthanded $(\mathrm{H}=-1)$ helical film, (b) 5-turns right-handed $(\mathrm{H}=+1)$ helical film, and (c) bilayer circular filter made of opposite structural handedness. 
helical structure is perpendicular to the substrate. The SEM micrograph of the bilayer circular filter also depicts the composition of two-layer helical film of opposite structural handednesses, as illustrated in Fig. 4 (c). The first layer deposited on the substrate has a right-handed structure and the second layer deposited on it has a left-handed structure. The thickness of each layer with 5 turns is $2.5 \mu \mathrm{m}$. Moreover, the SEM images also reveal that the $\mathrm{TiO}_{2}$ helical film and the bilayer circular filter deposited at $60^{\circ}$ are relatively closed-packed helical columns, indicating a minimal contribution from scattering in this regime.

\section{CONCLUSION}

We investigate the optical and structural properties of the $\mathrm{TiO}_{2}$ helical films of different structural handednesses and the bilayer circular filter of opposite structural handednesses fabricated by electron-beam evaporation via the GLAD technique. The results show that the $\mathrm{TiO}_{2}$ helical films of all structural handednesses display the circular Bragg phenomenon and the bilayer circular filter reflects both circular polarized lights (LCP \& RCP) with wavelength lying in the Bragg regime due to opposite structural handednesses. Also the analysis of SEM micrographs for the helical films and the bilayer circular filter shows relatively closed-packed helical columns, indicating a minimal contribution from scattering in this regime.

\section{ACKNOWLEDGMENT}

This work was supported in part by the Korea Science and Engineering Foundation (KOSEF) grant funded by the Korea government (MEST) (Quntum Photonic Science Research Center) and the Ministry of Knowledge Economy (MKE) and Korea Industrial Technology Foundation (KOTEF) through the Human Resource Training Project for Strategic Technology.

\section{REFERENCES}

1. A. Lakhtakia and R. Messier, Sculptured Thin Films: Nanoengineered Morphology and Optics (SPIE Press, Bellingham, WA, USA, 2005).

2. N. O. Young and J. Kowal, "Optically active fluorite films," Nature (London) 183, 104-105 (1959).

3. I. Hodgkinson and Q. H. Wu, "Inorganic chiral optical materials," Adv. Mater. 13, 889-897 (2001).

4. S. M. Pursel, M. W. Horn, M. C. Demirel, and A. Lakhtakia, "Growth of sculptured polymer submicronwire assemblies by vapor deposition," Polymer Com. 46, 9544-9548 (2005).

5. R. Messier, V. C. Venugopal, and P. D. Sunal, "Origin and evolution of sculptured thin films," J. Vac. Sci. Technol. A 18, 1538-1545 (2000).

6. B. Dick, M. J. Brett, and T. Smy, "Controlled growth of periodic pillars by glancing angle deposition,” J. Vac. Sci. Technol. B 21, 23-28 (2003).

7. K. Robbie, M. J. Brett, and A. Lakhtakia, "First thin film realization of a helicoidal bianisotropic medium," J. Vac. Sci. Technol. A 13, 2991-2993 (1995).

8. S.-H. Woo and C. K. Hwangbo, "Optical anisotropy of microstructure-controlled $\mathrm{TiO}_{2}$ films fabricated by glancing-angle deposition (GLAD), J. Korean Phys. Soc. 48, 1199-1204 (2006).

9. S. R. Kennedy, M. J. Brett, O. Toader, and S. John, "Fabrication of tetragonal square spiral photonic crystal," Nano Lett. 2, 59-62 (2002).

10. S. R. Kennedy and M. J. Brett, “Advanced techniques for the fabrication of square spiral photonic crystals by glancing angle deposition,” J. Vac. Sci. Technol. B 22, 1184-1190 (2004).

11. Y. P. Zhao, D. X. Ye, P. I. Wang, G. C. Wang, and T. M. Lu, "Fabrication of Si nanocolumns and Si square spirals on self-assembled monolayer colloid substrates," Int. J. Nanosci. 1, 87-97 (2002).

12. I. Hodgkinson and Q. H. Wu, "Birefringent thin-film polarizers for use at normal incidence and with planar technologies,” Appl. Phys. Lett. 74, 1794-1796 (1999).

13. A. C. van Popta, M. H. Hawkeye, J. C. Sit, and M. J. Brett, "Gradient-index narrow-bandpass filter fabricated with glancing-angle deposition,” Opt. Lett. 29, 2545-2547 (2004).

14. K. Kaminska, T. Brown, G. Beydaghyan, and K. Robbie, "Vacuum evaporated porous silicon photonic interference filters," Appl. Opt. 42, 4212-4219 (2003).

15. S. R. Kennedy and M. J. Brett, "Porous broadband antireflection coating by glancing angle deposition," Appl. Opt. 42, 4573-4579 (2003).

16. S.-H. Woo, Y. J. Park, D. H. Chang, K. A. Sobahan, and C. K. Hwangbo, "Wideband antireflection coatings of porous $\mathrm{MgF}_{2}$ films by using glancing angle deposition," J. Korean Phys. Soc. 51, 1501-1506 (2007).

17. Q. H. Wu, L. D. Silva, M. Arnold, I. J. Hodgkinson, and E. Takeuchi, "All-silicon polarizing filters for nearinfrared wavelengths,” J. Appl. Phys. 95, 402-404 (2004).

18. J. J. Steel, J. P. Gospodyn, J. C. Sit, and M. J. Brett, "Impact of morphology on high-speed humidity sensor performance," IEEE Sensors J. 6, 24-27 (2006).

19. J. J. Steel, A. C. van Popta, M. M. Hawkeye, J. C. Sit, and M. J. Brett, "Nanostructured gradient index optical filter for high-speed humidity sensing," Sensors and Actuators B 120, 213-219 (2006).

20. A. Lakhtakia and M. W. McCall, "Simple expressions for Bragg reflection from axially excited chiral sculptured thin films," J. Mod. Opt. 49, 1525-1535 (2002).

21. J. Adams, W. Haas, and J. Dailey, "Cholesteric films as optical filters,” J. Appl. Phys. 42, 4096-4098 (1971).

22. A. Lakhtakia, "Axial excitation of tightly interlaced chiral sculptured thin films: "averaged” circular Bragg phenomenon,” Optik 112, 119-124 (2001).

23. J. B. Sorage, A. C. van Popta, J. C. Sit, and M. J. Brett, "Circular birefringence dependence on chiral film porosity,” Opt. Exp. 14, 10550-10557 (2006). 
24. K. Robbie, L. J. Friedrich, S. K. Dew, T. Smy, and M. J. Brett, "Fabrication of thin films with highly porous microstructures,” J. Vac. Sci. Technol. A 13, 1032-1035 (1995).

25. K. Robbie and M. Brett, "Sculptured thin films and glancing angle deposition: growth mechanics and applications," J. Vac. Sci. Technol. A 15, 1460-1465 (1997).

26. A. C. van Popta, J. C. Sit, and M. J. Brett, "Optical properties of porous helical thin films,” Appl. Opt. 43, 3632-3639 (2004).

27. I. Hodgkinson, Q. H. Wu, B. Knight, A. Lakhtakia, and
K. Robbie, "Vacuum deposition of chiral sculptured thin films with high optical activity," Appl. Opt. 39, 642-649 (2000).

28. K. Kaminska and K. Robbie, "Birefringent omnidirectional reflector,” Appl. Opt. 43, 1570-1576 (2004).

29. Y. J. Park, K. A. Sobahan, and C. K. Hwangbo, "Wideband circular polarization reflector fabricated by glancing angle deposition,” Opt. Exp. 16, 5186-5192 (2008).

30. Y. J. Park, K. A. Sobahan, and C. K. Hwangbo, "Optical and structural properties of helical $\mathrm{TiO}_{2}$ films deposited by glancing angle deposition technique," J. Korean Phys. Soc. 52, S8-S12 (2008). 